\title{
The Neuroprotective Effect of Human CD 34 Positive Stem Cells on the Survival of Injured Retinal Ganglion Cells
}

\author{
Amro Ali*, Joyce Mbiziwo Tiapo, Thaddeus L Wandel and Sansar \\ Sharma \\ Department of Ophthalmology, New York Medical College, USA
}

Research Article

Volume 2 Issue 4

Received Date: November 07, 2017

Published Date: December 15, 2017

*Corresponding author: Amro Ali, MD, Department of Ophthalmology, New York Medical College, 40 Sunshine Cottage Road, Valhalla, NY 10595, USA, E-mail: amro.ali9@icloud.com

\section{Abstract}

Purpose: To assess the neuroprotective effect of CD 34+ stem cells on retinal ganglion cell (RGC) survival after optic nerve compression injury (ONCI).

Methods: The optic nerve compression injury was obtained through exerting pressure on the optic nerve $1 \mathrm{~mm}$ behind the eye globe for duration of 60 seconds. This compression was conducted using a stereotactic device attached to small forceps after good exposure to optic nerve. Treatment of ONCI model was done through intravenous administration of $3,000,000$ CD34+ stem cells intravenous through rat tail was done six hours after the onset of the nerve crush injury. Evaluation of the effect of CD34 positive stem cells on injured retinal cells was obtained 6 days after ONCI through the following: (1) immunohistochemistry through Brn-3b labelled RGCs and, (2) electrophysiological changes was studied using F-VEPS after dark adaptation period.

Results: Brn-3b labeled RGCs density was found with an average of $1490 / 70074 \mu^{2}$ in the normal retina of adult female Wistar rat animal, in compared to $366 / 70074 \mu \mathrm{m}^{2}$ in the retina of non treated ONCI. Flash-Visual Evoked potentials (f-VEPs) were prolonged from $50 \mathrm{msec}$ in control rats to $110 \mathrm{msec}$ in experimental rats after treatment with stem cells, there was an increase in the number of RGCs with an average of $999 / 70074 \mu \mathrm{m}^{2}$ and reduction in the latency of F. VEPs to $91 \mathrm{msec}$ in the CD 34 + treated group.

Conclusion: Intravenous administration of 3,000,000 human umbilical cord blood CD 34+ stem cells may have a potential neuroprotective effect on damage/injured retinal ganglion cells manifested by an increase in the survival of retinal ganglion cells (56\%)and an improvement in the latency period of f-VEPs in the optic nerve compression injury model treated with stem cells (33\%).

Keywords: Neuroprotective; Immunohistochemistry; Stereotactic; Canthatomy 


\section{Open Access Journal of Ophthalmology}

Abbreviations: ONCI: Optic Nerve Compression Injury; RGC: Retinal Ganglion Cell; IOP: Intraocular Pressure; SCI: Spinal Cord Injury

\section{Introduction}

World Health Organization statistics indicates that glaucoma accounts for blindness of at least 5 million people, causing $13.5 \%$ world blindness. In unites States, glaucoma is the second leading cause of blindness and the second most frequent reason for ambulatory visits to physician in US. In glaucoma, progressive death of Retinal Ganglion Cells (RGCs) leads to optic nerve degeneration and progressive loss of vision. The main lines of glaucoma treatment focus on reduction of intraocular pressure (IOP) either surgically or pharmacologically. There are strong evidences that lowering IOP provides neuroprotective effects on injured RGCs. Even with a controlled IOP, there is a progressive loss of RGCs. Consequently, there is an increase need for neuroprotective agents that may preserver RGCs in the presence of normal IOP in glaucoma patients.

The optic nerve compression injury (ONCI) has served as an important model for studying the non-neuronal response to an injury and for studying axon regeneration in the CNS [1-4]. It is also a well-established model to study glaucoma and RGCs. ONCI leads to death of RGCs as a direct result of primary damage to the axons secondary to the disruption of retrograde axonal transport [5]. Additional damage is caused by "secondary degeneration" of RGCs that escaped direct injury, but are susceptible to damage by different toxic mediators released into the extracellular environment by dying RGCs.

We focused in this experiment on restoring injured RGCs after ONCI through the use of Human umbilical cord blood stem cells (hUCBCSs). hUCBCSs are one of the strongest candidates used in cell therapy for spinal cord injury (SCI) because of its greater availability, weak immunogenicity, and lower risk of viral transmission [6]. The goal of this study is investigate the neuroprotective effects of hUCBCSsCD34+on adult rat after optic nerve compression injury. The neuroprotective effects of hUCBCSsCD34+ were assessed based on survival of RGCs and improvement in the latency time of F-VEPs.

\section{Material and Methods}

All experiments were performed in accordance with the ARVO Statement for the Use of Animals in Ophthalmic and Vision Research, and Animal Care and Use Committee requirements. In this study, we used adult female Wistar rat animals, as they have been known to be sensitive to optic nerve trauma based on the level of Glutamate mRNA $[7,8]$. In addition, Wistar rat animals have been used extensively in experimental ophthalmology and visual science for evaluation of different lines of treatment.

In this study, we aimed to evaluate the neuroprotective effect of hUCBCSsCD34+oninjured RGCs after optic nerve compression injury. We compared the neuroprotective effect of hUCBCSsCD34+on three different groups of animals:

(i) Group A, normal animals without any ONCI.

(ii) Group B, animals exposed to ONCI for one minute without stem cell therapy.

(iii) Group C, animals who were exposed to ONCI for one minute then treated for six hours after the onset of compression injury with 3,000,000 hUCBCSsCD34+.

The evaluation was conducted using immunohistochemistry to assess RGCs densities and the functional analysis we used electrophysiological function, f-VEPs, to measure the latency of flash visual evoked potential.

\section{Optic Nerve Compression Injury Device}

Our group built a simple device to provide a uniform amount of optic nerve damage. This optic nerve compression injury device was composed of blunt forceps attached and manipulated by a stereotaxic device manipulator. This model provided a more uniform amount of pressure on optic nerve rather than other maneuvers. After good exposure of the optic nerve through fine dissection of the surrounding tissues, the forceps was applied for one minute directly between the meanings and the optic nerve just one mm behind the eye globe to avoid injury of the capillary network. The micromanipulator was adjusted to enable the forceps to crush the optic nerve without any change in the amount of force-applied force exerted on the optic nerve [9]. This model was used to simulate mechanical non-ischemic trauma to the optic nerve.

\section{Optic Nerve Compression Injury}

Adult female Wistar rats were used with an average weight of 200-250 gm and average age of 2 to 3 months. The animals were anesthetized with $10 \mathrm{mg} / \mathrm{Kg}$ Xylazine (Phoenix Pharmaceutical, St. Joseph, MO) and $80 \mathrm{mg}$ Ketamin (Pfizer Parke-Davis, Morris Plain, NJ). Sterilization of the surgical field was done using Betadine. Topical anesthetic drops were used to the cornea five minutes before the surgery using $0.5 \%$ proparacaine 


\section{Open Access Journal of Ophthalmology}

(Alcaine, Ophthetic, Paracaine). The head was fixed in a stereotaxic frame and the surgical procedure was conducted through obtaining a good exposure of the optic nerve with minimum soft tissue damage and without injury to the surrounding blood vessels. We accessed the orbit through an incision in the lateral angle of the eye, a lateral canthatomy. The conjunctiva was dissected along the temporal limbus from 1 to 4 o'clock.

The eye globe was rotated nasally using a fixating suture (7-0 silk) through the conjunctiva and almost the entire Harderian gland was removed. While dislocating the eyeball anteriorly, the ocular muscles overlying the optic nerve was separated and held apart. The optic nerve was exposed by cutting the lateral rectus muscle and by blunt dissection of the tissue surrounding the optic nerve. Then with the aid of fine blunt scissors (used for tenotomy, Westcott), the tissues behind the eyeball and the extra ocular muscles around the optic nerve was dissected: the whole meninges were finely dissected along the optic nerve, while avoiding injury to the ophthalmic blood vessels. At this point, we had a good exposed portion of the optic nerve [10]. The meninges were cut sparing the surrounding blood vessels to have a good portion of the optic nerve. The compression injury was conducted $1 \mathrm{~mm}$ behind the eye globe for 60 seconds using the standard surgical forceps.

The skin and the conjunctiva were sutured using 4-0 silk and 7-0 nylon, respectively. Postoperative care consisted of the following; (i) topical antibiotics: Ocuflox (Of loxacine) 0.3\% 1-2 drops, once or as needed: (ii) topical anesthetics: proparacaine $\mathrm{HCl} 0.5 \%$ 1-2 drops, once or as needed: (iii) Carboxyl Methylcellulose: refresh celluvisc $1 \%$ once or as needed. We monitored the eye daily after the surgery to assess any signs of infection or rejection after hUCBCSsCD34 implantation such as corneal opacity, redness or intraocular hemorrhage.

\section{Immunohistochemistry}

Brn-3b is a member of the POU-domain family of transcription factors that plays a critical role in regulating gene expression, particularly in cells of the nervous system [11]. Brn-3b has been found to be expressed in a sub-population of the RGCs nuclei of various species, including mouse, chicken, rabbit, monkey and human [12].

\section{Brn-3b Stained Retinal Ganglion Cell Positive Nuclei}

Eyes were harvested from euthanized female Wistar rat and were dissected from the cornea, iris and lens.
Eyecups were rinsed in saline before fixation. The whole eyes were fixed for at least two hours in $4 \%$ Paraformaldhyde in Dulbecco's phosphate buffered saline (Invitrogen 14200-075, Grand Island, USA). Eyes were transferred to tris buffered saline with Tween-20 (TBST) (Dako Cytomation S3306, Carpentaria, CA, USA) and dissected to obtain intact, flattened retina in a four-leaf clover shape. Vitreous was disrupt ted and removed manually by pinching and tearing vitreous using two pair of fine forceps. The retina was immersed in TBST in a 24 well tissue culture plate (Costar, Corning, NY, USA) and was washed with successive rinse/drain cycles of TBST to remove the residual fixatives. Retina was permiabilized by 30-45 minutes incubation in $0.3 \%$ Triton X-100(X-100PC Sigma) in TBS, and then was washed three times with TBST to remove the detergent. Non specific binding of antibodies to resident retinal proteins were blocked by incubating the retina with $3 \%$ bovine serum albumin (BSA) (A-7030 Sigma) plus 10\% normal donkey serum (017-000-121 Jackson Immuno- Research) in TBST for 30 minutes with gentle agitation. If amplification of the BRN$3 \mathrm{~b}$ signal was desired, the endogenous retinal biotin (Bhattacharjee et al; Ruggiero and Sheffield) was also blocked using Avidin Biotin Block Kit (Vector Burlington, VT, USA) [11].

The retina was incubated overnight with goat antimouse Brn 3b (SC6026 Santa Cruz Bio-technology, Santa Cruz, CA, USA) and $3 \%$ BSA-TBST $(1: 50)$ at $4{ }^{\circ} \mathrm{C}$ with shaking balance, then wash was done using TBST for 15 minutes with gentle agitation in 24 wheels. Retina was then incubated with 1:200 dilution of donkey-anti-goat biotin (705-065-1470) for two hours with gentle agitation. Again, another wash was done using TBST for 15 minutes with gentle agitation in 24 wheels.

Amplification was obtained using a streptovidien Alexa 594 (5-11227) molecular probe in TBST-3\%BSA (1:400) for 30 minutes with gentle agitation. All retinal tissue was flattened on super frost plus microscope slide 12-550-15 (Fischer Scientific, Pittsburgh, PA). Then mounting was done using an Anti Fade Kit (P-7481 Molecular Probs). Cover slides were added on the retinal tissue. After the slide dried, four areas circling the optic nerve from each retina were digitally imaged. We used a DAS Microscope LEICA DM IRB/E, which was mounted by SPOT RT color camera. ADVANCED SPOT software was used for image adjustment [13].

\section{Image Capture and Analysis}

The images were obtained using fluorescent microscopy (DAS Microscope LEICA DM IRB/E, which was 


\section{Open Access Journal of Ophthalmology}

mounted by SPOT RT color camera. ADVANCED SPOT software was used for image adjustment) by obtaining four different images in four different quadrants with the same distance from the optic disc to assure a uniform density of RGCs. Capturing of these images were obtained using 10X for better localization of the optic disc, then we had four different images in four different quadrants with an equal distance from the optic nerve to ensure the homogenous RGCs density using 40X lens power. Processing of these images were conducted using Spot Advanced Medicine Software (SPOT Imaging Solutions, a division of Diagnostic Instruments, Inc., 6540 Burroughs Sterling Heights, MI 48314, USA).

The images analysis was conducted using NIH-Soft image analysis $\mathrm{J}-1.33$, which allowed us to analyze the fluorescent particle with an average size of 20-60 $\mu \mathrm{m}$ square ( 2 to 6 pixels). The analyses of these images were done using the fast red fluorescent mode. This was started with subtraction of the background with enhancing of the contrast, which was followed by adjusting the threshold. Finally, the particles analysis was obtained with an average size of 2 to 6 pixels. The chosen four quadrants surface area is $700074 \mathrm{um}^{2}$ in comparison to the surface area of the whole retina of rat, which is $3.8 \mathrm{~mm}^{2}$.

\section{Flash Visual Evoked Potential (f-VEPs)}

F-VEP is a reliable method for testing the integrity of the visual pathway from the retina to cortex. The rats were deeply anesthetized with sodium pentobarbital (50 $\mathrm{mg} / \mathrm{Kg}$ ) and placed into a stereotactic head frame device. Two holes were drilled through the skull of each animal, enabling two stainless steel frame screws to be placed in contact with the cortex serving as electrodes. The dura was kept intact to minimize the cortical damage. The "reference" and" active" electrodes positions were $5 \mathrm{~mm}$ anterior to the bregma, $1.5 \mathrm{~mm}$ left of the midline, and 8 $\mathrm{mm}$ caudal to the bregma and $3 \mathrm{~mm}$ right of the midline, respectively.

F-VEP originates from the left eye, recorded in the right visual cortex, and was evoked by light stimulation after implantation of the electrodes. The xenon light source of a Grass PS22C Photic Stimulator was centered over the left eye at a distance of $10 \mathrm{~cm}$, while the right eye was covered with black plastic tape to avoid the possibility of a VEP response originating from it. Single flash stimuli $10 \mathrm{~m} \mathrm{sec}$ in duration were delivered in darkened room at an interstimulus interval of approximately $10 \mathrm{sec}$ with a peak light intensity of 19 million lumens. The signal from the reference and active electrode were differently amplified at a again of 1000, filtered between 1 and $100 \mathrm{~Hz}$, and sampled digitally at $500 \mathrm{~Hz}$.

F-VEPs measurements were recorded as a voltage fluctuation during a single 1000-msec sweep, where the beginning of each sweep coincided with triggering of light stimulus. Minimum of 60 individual measurements were collected and were presented as mean plus or minus standard of deviation. F-VEPs were done 6 days after optic nerve crush injury to the three groups of animals. These animal groups included the followings: (i) normal rats without crush injury, (ii) Sham control, non stem cells treated ONCI rats; and (iii) hUCBCSsCD34+treated ONCI rats.

\section{Stem Cell Isolation}

Endothelial Progenitor Cells: Fresh whole blood was obtained from the human cord blood under an IRB approved protocol. Blood was collected in heparinized tubes. The blood was diluted 1:2 in phosphate buffered a line (PBS) plus $2 \mathrm{mM}$ EDTA (Ethylene diaminetetra acetic acid), layered onto lymphocyte separation medium (Ficoll, density $1.077 \mathrm{~g} / \mathrm{ml}$, ICN Biomedical, Aurora, OH) and centrifuged for 30 minutes at 1,900 RPM, and at a temperature of $20^{\circ} \mathrm{C}(35 \mathrm{ml}$ blood was very gently added onto $15 \mathrm{ml}$ lymphocyte separation medium in every $50 \mathrm{ml}$ sterile tube). Then the white ring fraction (mononuclear cell layer) was transferred to a new $50 \mathrm{ml}$ tube using a sterile Pasteur pipette, PBS was added two times and the tubes were centrifuged for 15 minutes at 1500 RPM at room temperature. After discarding the supernatant, the pellet was resuspended in $4 \mathrm{ml}$ ACKlysine buffer to get rid of (for lysing) remaining erythrocytes. Mononuclear cells (MNCs) were incubated in the ACK lysine buffer for no more than 3 minutes on ice. After 3 minutes, $20 \mathrm{ml}$ PBS was added to the solution, the cells were washed twice (centrifuge at 1200 RPM) with PBS.

Mononuclear cells (MNCs) were subjected to immunomagnetic separation using an AC133 Cell Isolation Kit (Miltenyi Biotech; Auburn, CA; http://www.miltenyibiotec.com), according to published methods $(45,46)$. Briefly, MNCs were incubated for 45 minuteson ice with an FcR-blocking reagent (human IgG) and AC 34MicroBeads. These micro beads are conjugated with monoclonal mouse anti-human antibodies against AC133. After washing with PBS plus 2 mM EDTA, the labeled cells were filtered through a $20-\mu \mathrm{m}$ nylon mesh and loaded onto a column that was installed in a magnetic field. Trapped cells were eluted after the column was removed from the magnet. The collected cells were named as AC 34+ endothelial progenitor cells. 


\section{Open Access Journal of Ophthalmology}

\section{Results}

\section{Immunohistochemistry}

Quantitative analysis of experimental animals was done for 6 rats 6 days after ONCI, two in each group. These rats showed no agitation or evidence of rejection following hUCBCSsCD34+transplantation. All rats survived the procedure including anesthesia, ONCI and stem cell intravenous administration without complications. Evaluation of the of the neuroprotective effects of hUCBCSsCD34+stem cell on survival of RGCs after optic nerve crush injury was studied by assessing Brn-3b labeled RGCs density in four different quadrants with the same distance from the optic nerve.

Brn-3b labeled RGCs density was $1490 / 70074 \mu \mathrm{m}$ in normal retina (Figures 1-3). In the sham-surgery group, Brn-3b labeled RGCs density was $366 / 70074 \mu \mathrm{m}$ (Figures 4-6), Brn-3b labeled RGCs density was $999 / 70074 \mu \mathrm{m}$ in the retina of hUCBCSsCD34+treated ONCI model (Figures $7,8,9)$. The number of RGCs was obviously reduced in the retina of the optic nerve crush injury model with an average of $366 / 70074 \mu \mathrm{m}$, with a reduction of almost $75 \%$ of RGCs of the normal retina. Brn-3b labeled RGCs density was obviously increased after systemic intravenous injection of 3,000,000 which was done 6 post-ONCI, with an average of $999 / 70074 \mu \mathrm{m}$ in the treated group, with an increase in the survival of damaged RGC at a mean count of $633 / 70074 \mu \mathrm{m}$, saving more than $56 \%$ of damaged RGCs.

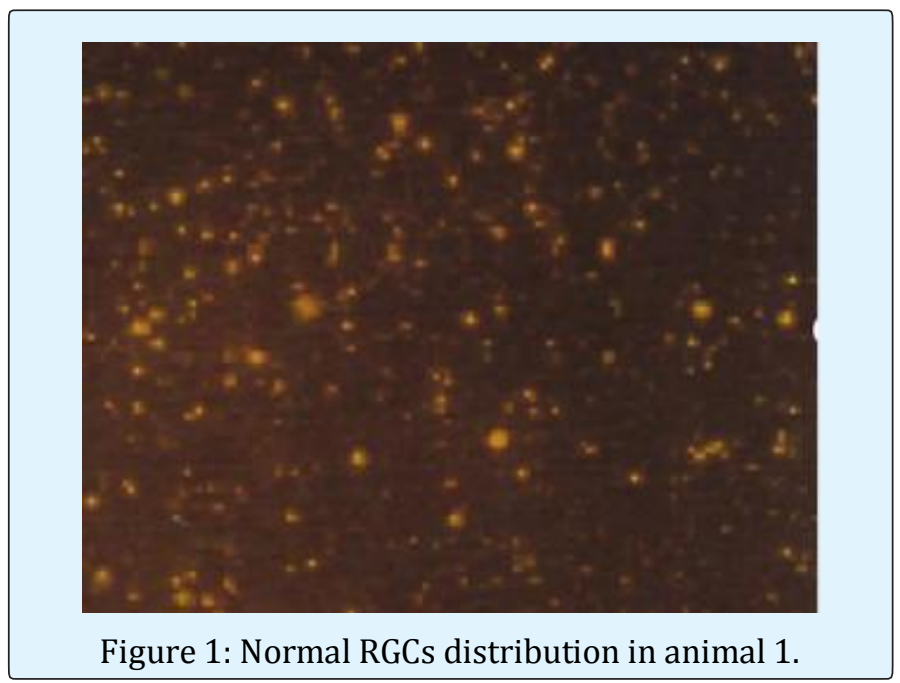

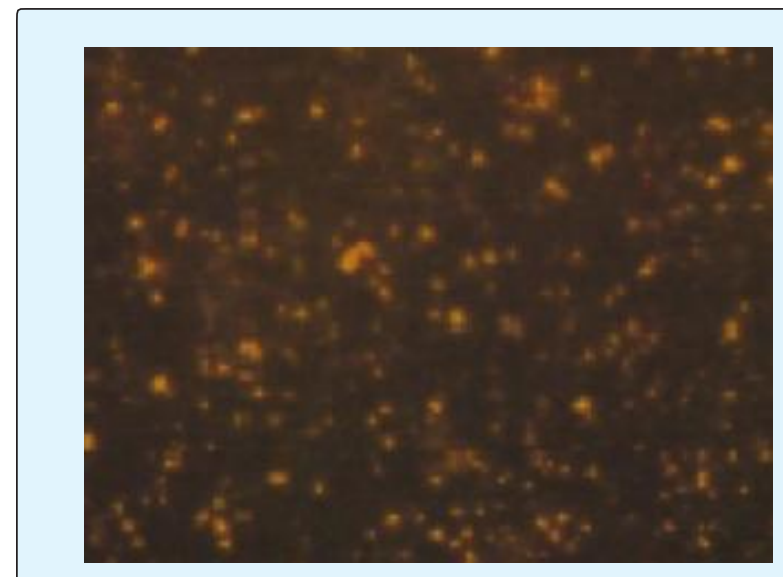

Figure 2: Normal RGCs distribution in animal 2.

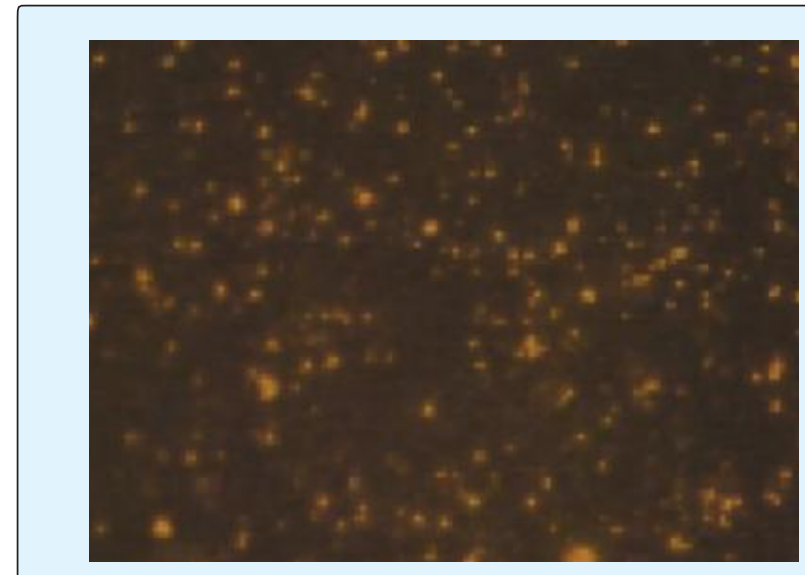

Figure 3: Normal RGCs distribution in animal 3.

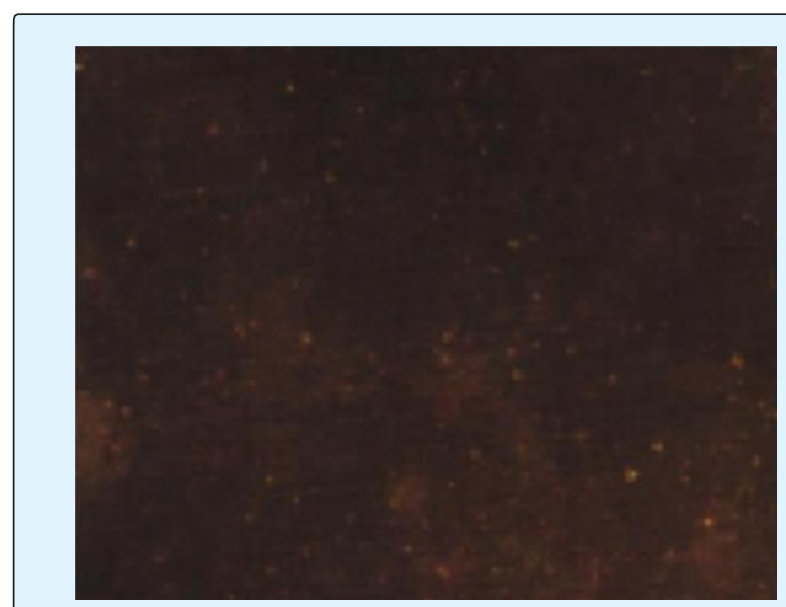

Figure 4: Optic Nerve Compression Injury in animal 4. 


\section{Open Access Journal of Ophthalmology}

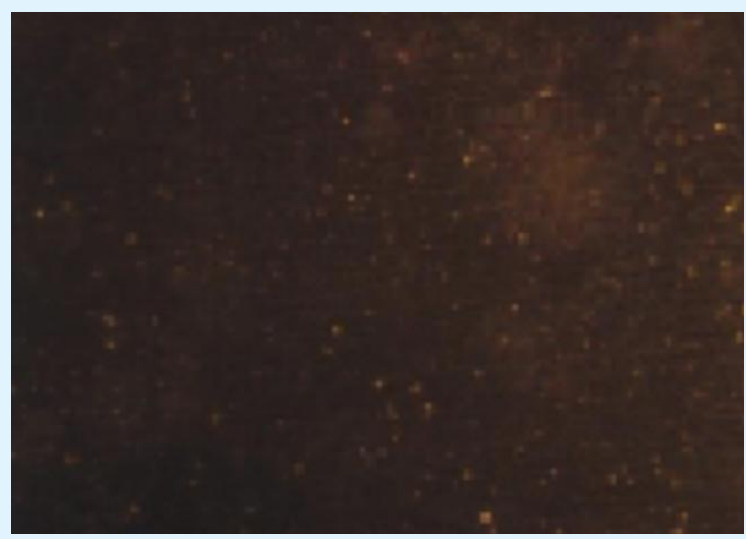

Figure 5: Optic Nerve Compression Injury in animal 5.

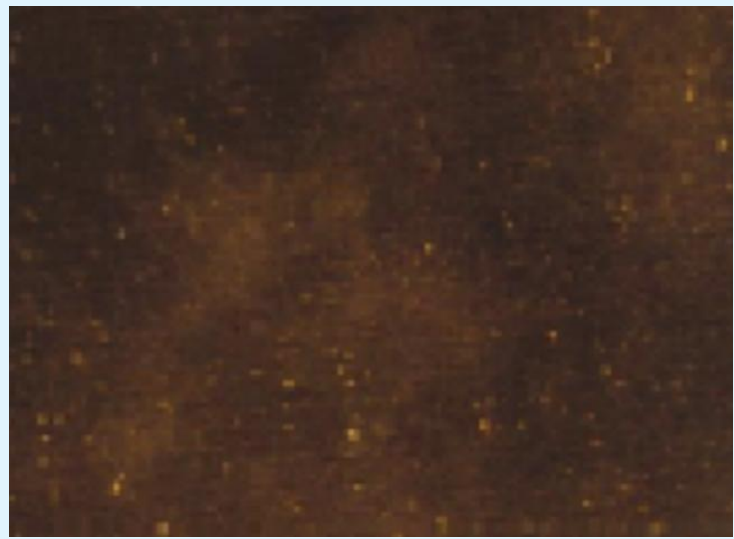

Figure 6: Optic Nerve Compression Injury in animal 6.

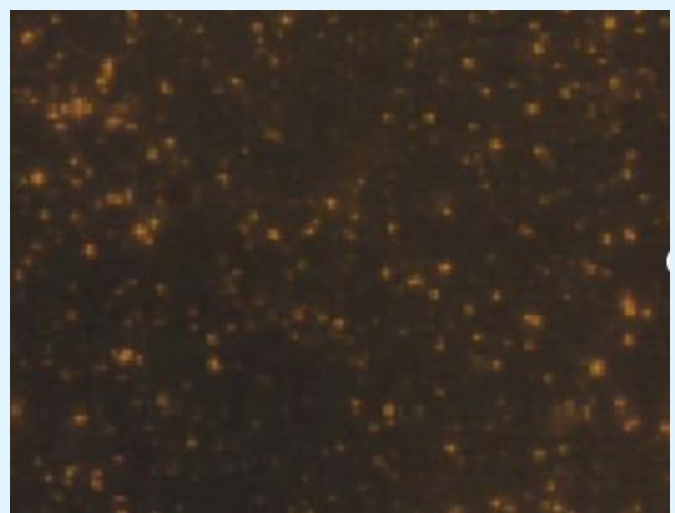

Figure 7: Optic Nerve Compression Injury treated with stem Cells in animal 7.

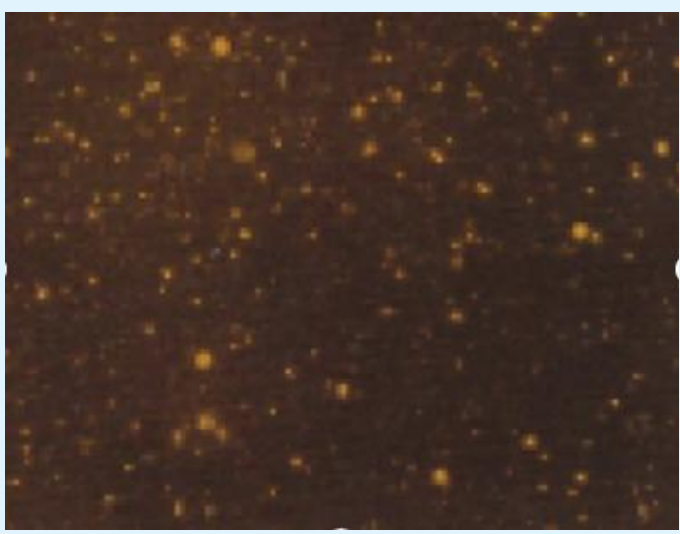

Figure 8: Optic Nerve Compression Injury treated with stem Cells in animal 8.

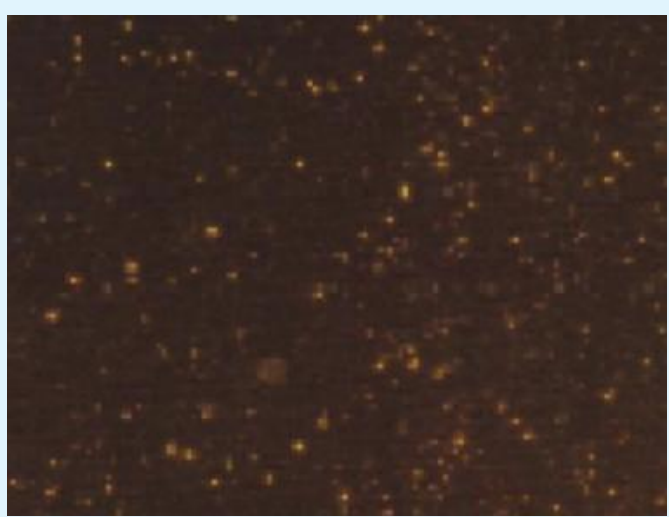

Figure 9: Optic Nerve Compression Injury treated with stem Cells in animal 9.

\section{Flash -Visual Evoked Potentials}

Flash -Visual Evoked Potentials was done for 6 rats 6 days after ONCI, two in each group. Comparison of F-VEPs latencies between non treated ONCI and the stem cells treated ONCI group in comparison to normal rats without ONCI:) and the latency period was $50 \pm 0.9 \mathrm{msec}$ (Figure 10) in normal rats. This result was compared to the the latency period of non treated ONCI model which was 110 $\pm 1.2 \mathrm{msec}$ (Figure 11) and to the latency period of hUCBCSsCD34+ treated ONCI, which was $90 \pm 1.1 \mathrm{msec}$ (Figure 12). This revealed prolonged latency after ONCI, which was partially shortened with hUCBCSsCD34+ transplantation. 


\section{Open Access Journal of Ophthalmology}
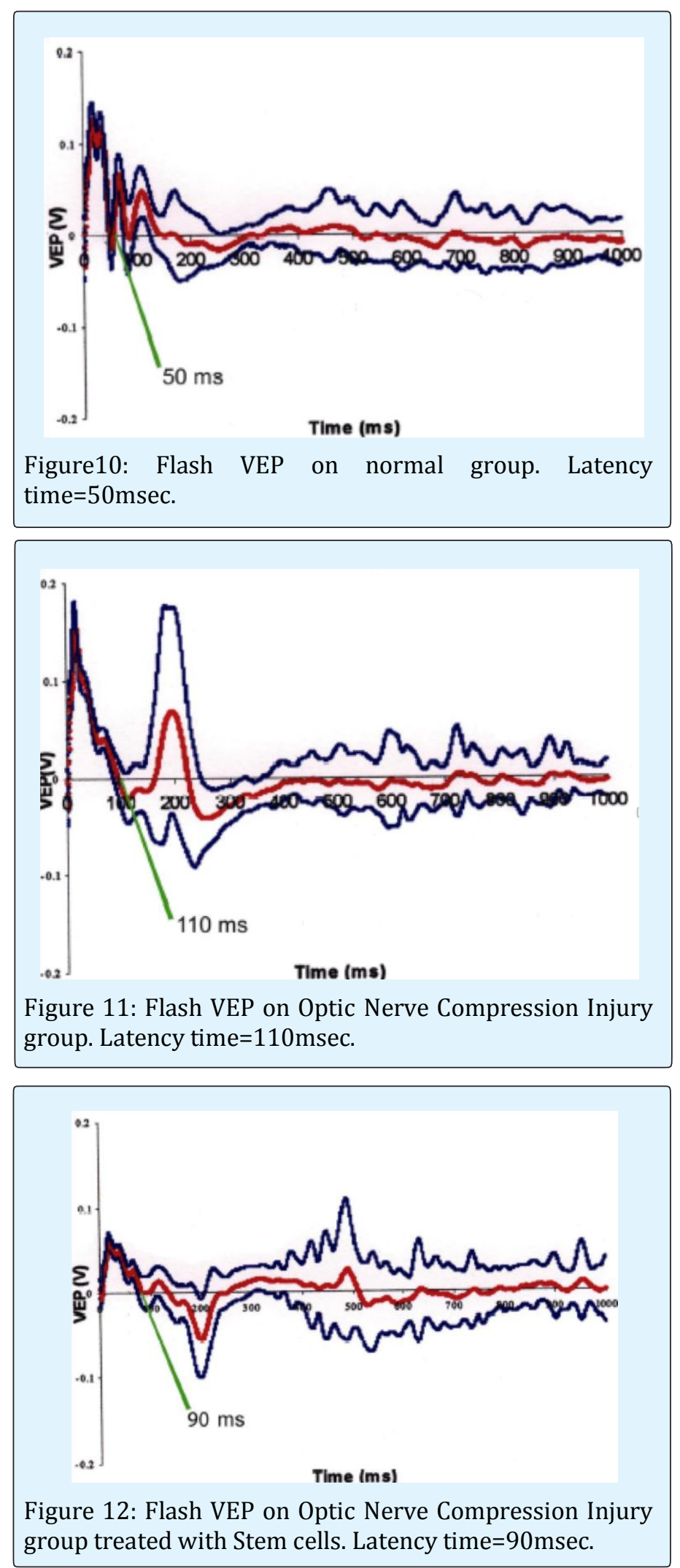

\section{Discussion}

We report for the first time that systemic intravenous administration of hUCBCs CD34+ have a neuroprotective effect on injured RGCs after ONCI: this effect was demonstrated by increasing the survival of retinal ganglion cells and improving the latency period of f-VEPs. In this study, optic nerve compression injury was accomplished using our previously described device (see methodology): this compression was occurred for 60 seconds through applying the forceps on the optic nerve 1 $\mathrm{mm}$ behind the eye globe. This compression injury to the axons of the neurons triggers degenerative responses. These degenerative reactions proceed retrograde to the soma and this is termed "Wallerian degeneration".

After induction of ONCI, we injected 3,000,000 hUCBCSsCD34+intravenously six hours after the onset of the crush injury. We performed Immunohistochemistry and electrophysiological studies days after ONCI to assess the neuroprotective effects. These neuroprotective effects could be attributed to the migration of the stem cells to the site of injury in response to chemotactic factors release, in this case the optic nerve trauma. The compression injury of the optic nerve leads to complement activation at the site of injury and along the distal portion of the nerve, as well as up regulation of the complement inhibitor Cluster in astrocytes. Reactive microglial cells seem to have a key role in complement activation as a local source of C3. It has been suggested that the balance between complement activation and their regulators may have an impact on axonal degeneration following opticnerve injury [14].

In this study, intravenous administration of 3,000, 000 hUCBSC CD34 + demonstrated neuroprotective effects on the optic nerve crush injury model presented with an increase in the survival of RGCs. Brn -3b labeled RGCs density was $1490 / 70074 \mu \mathrm{m}$ in the normal retina in comparison to $366 / 70074 \mu \mathrm{m}$ in the retina of non treated ONCI model. There was a loss of an average 1124 / $70074 \mu \mathrm{m}$. In the CD 34+ treated group, Brn-3b labeled RGCs density was only reduced to 999 / 70074 in comparison to the non-treated group where the average of RGCs was $491 / 70074 \mu \mathrm{m}$. Thus, immunohistochemistry study provides credence to the idea that CD34+ saved more than $505 / 70074 \mu \mathrm{m}$.

The average latency period of F-VEPs was $50 \mathrm{msec}$ in normal retina in comparison to non-treated optic nerve compression injury at $110.6 \mathrm{msec}$. There was an improvement in the latency period of f-VEPS in the optic nerve compression injury model, which was treated with 


\section{Open Access Journal of Ophthalmology}

3,000,000 CD 34+ stem cells with an average latency period of $91 \mathrm{msec}$, in comparison to the other ONCI nontreated group with an average latency period of 110.6 msec. As such, CD 34+ stem cell intravenous administration improved the conductivity in the visual pathway and presented with a shortening of the latency period by $19.6 \mathrm{msec}$.

The improvement in RGC survival and in f-VEPs could be attributed to neuroprotection rather than neurodifferentiation due to difficulty in stem cell penetration of Bruch's membrane. This study demonstrated the neuroprotective effect of stem cells but did not reveal their mechanisms. We will continue our work in this area exploring the underlying mechanism of neuro enhancement and neuroprotection. We will study the role of different neurotrophic and growth factors in neuroprotection.

Previous studies demonstrated the neuroprotective effects of neurotrophins on the development and the plasticity of the visual system of mammals and in particular the visual cortex. In addition, NGF was found to prevent all effects of monocular deprivation [15]. These neurotrophins: NGF, BDNF, NT3, and N4 have a wide range of effects in the development and the regeneration of the neuronal circuit of the visual system.

This study has few pitfalls, such as the small number of animals, absence of a positive control, and incomplete analysis of f-VEPs and lack of study on neuroprotective effect mechanisms through assessment of different neurotrophic factors. We will address these shortcomings in our future work.

This is a preliminary, but a promising experiment where we explored the complicated physiology of the neural circuit and how much hUCBCSsCD34+will have a contributing role in the neuroprotection of RGCs in compression optic neuropathy. Further work is required to assess the progression of apoptosis and if the presence of stem cell will delay or stop this process, progression of apoptosis. This will include assessment of different growth and neurotrophic factors levels such as BDNF, NGF, NT3, NT4/5, CNTF, GDNF in addition to neurotrophins' surface receptors such as TrKA, Tropomyosin related kinase family and the $\mathrm{p} 75$ receptors. RGC die by apoptosis and this process were demonstrated in models of optic nerve compression injury or axotomy, experimental glaucoma and in human glaucoma. This apoptotic process is triggered by various stimuli. It involves intrinsic and extrinsic pathways which are the major area of research to prevent or delay RGC death in glaucoma (Molecular basis).

\section{Summary}

We have demonstrated that intravenous administration of 3,000,000 hUCBCSsCD+ 34 stem cells have a significant impact on preserving of injured RGCs and retaining their electrophysiological functions. We found that 3,000,000 hUCBCSs CD+ 34 intravenous administrations preserve up to $63 \%$ of injured cells and improve latency time by $33 \%$.

Conflicting Interest: There are no conflicts of interest to disclose.

\section{References}

1. Berry MJ, Carlile J, Hunter A (1996) Peripheral nerve explants grafted into the vitreous body of the eye promote the regeneration of retinal ganglion cell axons severed in the optic nerve. J Neurocytol 25(2): 147-170.

2. Fournier AE, McKerracher L (1997) Expression of specific tubulin isotypes increases during regeneration of injured CNS neurons, but not after the application of brain-derived neurotrophic factor (BDNF). J Neuroscience 17(12): 4623-4632.

3. Vidal Sanz M, Bray GM, Villegas-Perez MP, Thanos S, Aguayo AJ (1987) Axonal regeneration and synapse formation in the superior colliculus by retinal ganglion cells in the adult ret. J Neuroscience 7(9): 2894-2909.

4. Villegas-Perez MP, Vidal-Sanz M, Bray GM, Aguayo AJ (1988) Influences of peripheral nerve grafts on the survival and regrowth of axotomized retinal ganglion cells in adult rats. J Neuroscience 8(1): 265-280.

5. Barron KD, Dingerer MP, Krohel G, Easton SK, Mankes R (1986) Qualitative and quantitative ultrastructural observations on retinal ganglion cell layer of rat after intraorbital optic nerve crush. J Neurocytology 15(3): 345-362.

6. Lewis ID (2002) Clinical and experimental uses of umbilical cord blood. Intern Med J 32(12): 601-609.

7. Zhao ZM, Li HJ, Liu HY, Lu SH, Yang RC, et al. (2004) Intraspinal transplantation of CD34+ human umbilical cord blood cells after spinal cord 


\section{Open Access Journal of Ophthalmology}

hemisection injury improves functional recovery in adult rats. Cell Transplant 13(2): 113-122.

8. Li Y, Schlamp CL, Nickells RW (1999) Experimental induction of retinal ganglion cell death in adult mice. Invest Ophthalmology Vis Sci 40(5): 1004-1008.

9. Mawrin C, Pap T, Pallas M, Dietzmann K, BehrensBaumann W, et al. (2003) Changes of retinal glutamate transporter GLT-1 mRNA levels following optic nerve damage. Mol Vis 9: 10-13.

10. Jiang B, Jiang Y (2001) A model of calibrated optic nerve crush injury in rats. Yan Ke Xue Bao 17(2): 99$102,121$.

11. Gellrich NC, Schimming R, Zerfowski M, Eysel UT (2002) Quantification of histological changes after calibrated crush of the intraorbital optic nerve in rats. Br J Ophthalmology 86(2): 233-237.
12. Latchman DS (1999) POU family transcription factors in the nervous system. J Cell Physiol 179(2): 126-133.

13. Xiang M, Zhou L, Macke JP, Yoshioka T, Hendry SH, et al. (1995) The Brn-3 family of POU-domain factors: primary structure, binding specificity, and expression in subsets of retinal ganglion cells and somatosensory neurons. J Neuroscience 15 (7): 4762-4785.

14. Leahy KM, Ornberg RL, Wang Y, Zhu Y, Gidday JM (2004) Quantitative ex vivo detection of rodent retinal ganglion cells by immunolabeling Brn-3b. Exp Eye Res 79(1): 131-140.

15. Ohlsson M, Bellander BM, Langmoen IA, Svensson M (2003) Complement activation following optic nerve crush in the adult rat. J Neurotrauma 20(9): 895-904. 